many months consecutively from two large issues on the nape of the neck.

Autopsy.-Nothing particular was found in the head, excepting that the arachnoid membrane over the pons varolii adhered to the parietal layer of that tissue, and about two ounces of serum were found in the ventricles; but there was no tumor or change of structure either in the brain or in the cellebellum. The thoracic and abdominal viscera were healthy, with the exception of the bladder, which was much contracted in size, thickened in its coats, whilst the omentum and some of the small intestines adhered to its surface. On opening the spinal canal, the theca, corresponding to the three or four lower cervical vertebræ, was much distended; the arachnoid cavity was filled with lymph, and there were adhesions of the membranes to the chord, which appeared firmer at the anterior than at the posterior portion, and the parts were inseparable. The chord itself was longer than usual at this particular point, felt soft and pulpy to the touch, and on being divided it was found to be in an almost diffluent state, infiltrated with serum, but of a natural color. For the extent of half an inch above this point the chord exhibited adusky red color, but there was no difference obscrvable betwixt the two columns, being alike softened and discolored; the parts above and below being perfectly healthy, and of a natural appearance.

\section{ACADEMY OF MEDICINE.}

\section{November 8, 1842.}

The great part of this and the preceding meetings was occupied by a discussion between M. Guérin and M. Bouvier on division of the flexor tendons of the hand.

\section{URINARY FISTULA.}

M. H. Larry communicated a very curious case of urinary fistula, which he had cured by operation. The subject of the case was a female who had been long subject to urinary fistula situate beneath the umbilicus; the disease originated in inflammation of an ovarian cyst, containing hair, which had communicated on one side with the bladder and on the other with the parietes of the abdomen. The contents of the cyst, composed of viscid and purulent matter, hairs, and calcareous concretions, had been discharged at various times from both orifices. At length a large calculus formed in the bladder, and, getting entangled in the opening of the ureter, had forced the urine to issue, almost constantly, through the fistulous orifice in the abdomen.

After considerable reflection, $M$. Larry determined on performing an operation founded on the principle of lithotomy, as practised above the pubes. With this object he divided the fistula freely inferiorly, ex. posed the cyst, extirpated a tumor to which adhered a long lock of hair and followed the latter into the bladder, where it formed the nucleus of the stone, which he removed. The immediate effects of this very formidable operation were comparatively mild, and the patient recovered perfectly.

\section{SHEFFIELD MEDICAL SOCIETY.}

$$
\text { Nov. 3, } 1842 .
$$

Mr. Ovenexd in the Chair.

$$
\text { SUSRECTED POISONING. }
$$

The subject announced for the evening's discussion was a paper by Dr. Favell on " certain morbid phenomena illustrative of the reflex function of nerves ;" but in consequence of two gentlemen having come from a distance in the country for the purpose of laying before the society the details of some cases of suspected poisoning, Dr. F. consented to postpone the reading of his paper till some future meeting.

All the cases occurred in the members of one family, and the following are the principal facts which were detailed:-About five weeks ago, three persons, out of a family of six, residing in a village in Derbyshire, dined off dumplings made from a stone of flour which had been purchased at a neighbouring shop, and which, before being used, had stood, for at least one whole day, uncovered on a table in the house. Very soon after dinner, those who had eaten were seized with violent pain in the epigastrium, vomiting, and purging. The disturbance in the bowels, however, was very much greater in two of the cases than in the third. The remaining three of the family, who were not at dinner, came home to tea, and ate of bread made of the same flour as the dumplings. They, too, were speedily seized with similar symptoms to the three former. The whole family was in perfect health before partaking of the dumplings or bread. The surgeon who was called in at first suspected that the whole had been poisoned by mushrooms. The consecutive symptoms, however, as well as the people's own statement, soon induced him to take a very different view. After the violence of the symptoms subsided, very formidable phenomena still remained. The whole continued to droop and waste away. The inferior extremities became, in a considerable degree, paralysed; the abdomen assumed a flattened appearance; the hand was partially powerless, and there was considerable pain through the ball of the thumb. In sixteen days the father of the family, aged fifty-seven, died, and was buried, unhappily, without any post-mortem examination being performed. The rest of the pationts, up to the present time, have gradually become worse. The emaciation is extreme, the prostration excessive, and the pulse remarkably rapid, beating in some about a hundred and sixty strokes in a minute. In some of the cases the diarrhœa still continues, whilst in others it has been succeeded by obstinate constipation. No tenderness has been discovered by a very accurate examination of the spinal column.

It is proper to remark that there have not been very often cases of sudden illness in the neighbourhood. The dumplings and the bread were cooked in iron pans, and the water which was mixed with the flour was taken from an open well, and is constantly used by the whole neighbourhood. The family deny having had anything poisonous in the house, and it appears that they are on good terms with their neighbours. In the confusion, when all were taken ill, the remnant of the stone of flour was lost sight of, and its history cannot now be traced. The whole of the baking of bread was eatcn at intervals, and in small quantities at a time, by the sick family. 
There naturally arose, from the foregoing details, two topics of inquiry-viz., first, whether the symptoms which have been described were occasioned by poison, and secondly, assuming poison to have been administered, what was it? These two questions were pretty fully discussed, and the members appeared to be unanimously of opinion that the symptoms were attributable to poison, and that arsenic most probably was the deleterious agent which had been administered.

Mr. H. Jackson afterwards exhibited a singular gorget, which was invented some years ago by a very eccentric surgeon, who declared that by the use of this instrument the operation of lithotomy would be rendered "safe, easy, and agreeable." It is not needful to describe the instrument, as it was never used even by the inventor. Mr. Jackson contrasted with it the ingenious gorget which was invented and always used by the late Mr. Stainforth, of Sheffield.

\section{NEWCASTLE-ON-TYNE INFIRMARY.} PLEURISY WITH EFFUSION-DEATH- ABSENCE OF A KIDNEY,

(Reported by Mr. Taylor, House-Surgeon.)

Samuel Hamilton, aged twenty-four, employed at an alkali manufactory, was admitted a patient of this infirmary, under Dr. Cargill, on April 14, 1842. His complexion was dark, the face pale and anxious ; he was emaciated, and had been ill and unable to work for six weeks; his principal complaint was of dyspnœa; had some pain of right side for a short period, but not acute; his intellect appears confused, and he is not able to give decided answers to ques. tions, so that a satisfactory account of his previous symptoms cannot be obtained. He has a troublesome cough, with viscid mucous expectoration; urgent dyspnœa, which has been gradually increasing; face covered with copious perspiration; pulse 120 , very small, and weak; great thirst, tongue dry; bowels costive; sense of weight in right chest, but no pain; can only lie with his body inclined to the right side; respiration very quick and laborious.

Physical Signs.-The right side of the chest measures one inch and a quarter more than the left; intercostal spaces nearly obliterated; liver rests below the level of false ribs ; universal dulness on percussion, except in the infraclavicular region, where it is less so ; no respiration audible anteriorly, except between third rib and clavicle, where it is very feeble and indistinct; posteriorly, bronchial respiration and bronchophony at the root of the lung; no vesicular murmur. Left side of chest: respiration in superior lobe normal; in lower lobe, accompanied by loud murmurs and sonorous rales. Ordered to be bled to sixteen ounces, and to have

Calomel, two grains ;

Opium, one-eighth of a grain, every third hour.

15. No relief; profuse perspiration; panting respiration.

Calomel, three grains :

Compound kino powder, four grains; to be taken every second hour. To have some strong mercurial ointment rubbed in along the thighs every fourth hour.

Paracentesis thorasis to be performed, which was done at three o'clock in the afternoon, and three half. pints of clear straw-colored fluid drawn off; on standing a few minutes, the fluid formed into a jelly, and was proved by the usual tests to be highly albuminous. In the evening he was something easier; respiration not so laborious or frequent; pulse improved.

16. Has been able to lie a little on the left side; pulse 120, fuller; respiration thirty-six in the minute; sputa viscid and streaked with blood; sub-crepitant râle and bronchial respiration in upper part of left lung; right side of chest remains dull on percussion, and no return of respiration; great thirst; tongue very dry; heat of skin. A large blister to the right side of the chest; bleeding to twelve ounces.

Tartar emetic, one-eighth of a grain;

Water, half an ounce. To be taken every third hour.

Continue pills and mercurial ointment.

17. Is much the same but rather weaker. To have fifty drops of tincture of opium at night. Continue other remedies.

18. No better; slept worse last night.

19. Breathes easier; cough less troublesome; pulse 108, very soft and flowing; tongue much furred; gums a little swollen; can lie better on right side; percussion clearer over sternum; no respiration audible on right side; mucous rale continues in left lung. Calomel pills to be omitted.

Continue mercurial ointment and tartar emetic draught. An opiate mixture at night.

21. Respiration continues somewhat easier, is 26 in the minute; pulse 90 , and very irregular; gums more swollen and tender; perspiration still profuse.

Sesquicarbonate of ammonia, ten grains;

Water, an ounce; to be taken every third hour. To have two glasses of wine daily.

Continue the ointment.

22. Is becoming weaker; pulse continues very weak and irregular; no relief of symptoms.

23. Died at half-past seven in the evening.

A post-mortem examination was made the next morning at eleven o'clock. The body was not much wasted; no anasarca; the right pleural cavity contained one gallon and a half of clear greenish yellow fluid, which, on standing, was converted into a pretty firm jelly; the whole of the costal pleura was lined with a layer of rather firm, pale, coagulated lymph, which was deposited in greatest quantity near the sternum and pericardium; the lung was compressed into very small bulk, and lay close to the spinal column, and hidden by the pericardium; there was no lymph on its pleura, and no disease in its substance ; the left pleura was quite healthy; no adhesions; the lung firmer than usual, of a dark color, crepitant, and contained some serum; the mucous membrane of the bronchial tubes much injected, and coated with reddish mucus; the large tubes contained a quantity of grey viscid mucus; the pericardium contained about one ounce of serum; the heart healthy, no blood in its cavities; the right auricle and ventricle filled with yellow coagula; the spleen very dark, and softening had taken place in its centre; the right kidney very large and lobulated; no kidney existed on the left side.

Before opening the chest a trocar was pushed into it to draw off the fluid, but none flowed, although it was tried in several places, both before and after opening the chest. 